\title{
Eficiência relativa na gestão dos recursos da educação: uma análise dos cursos de formação inicial e continuada no Instituto Federal de Educação, Ciência e Tecnologia da Bahia
}

\begin{abstract}
Relative effectivenenss in the management of education resources: an analyisys of the initial and continued formation at Instituto Federal de Educação, Ciência e Tecnologia da Bahia
\end{abstract}

Efficacité relative dans la gestion des ressources éducatives: analyse de la formation initiale et continue de Instituto Federal de Educação, Ciência e Tecnologia da Bahia

Fábio Lemos Mota ${ }^{1}$

Universidade Federal da Bahia

Roberto Brazileiro Paixão ${ }^{2}$

Universidade Federal da Bahia

Adriana Omena Santos ${ }^{3}$

Universidade Federal de Uberlândia

Resumo: Foi analisado a eficiência relativa dos campi do IFBA como principal objetivo desta pesquisa. Além de definir as eficiências em oferecer cursos de formação inicial e continuada (FIC) com os recursos disponíveis e fazer recomendações para a melhoria da eficiência das unidades analisadas. O método de análise empregado é o quantitativo, com o uso da técnica de Análise Envoltória de Dados. Analisa apenas 6 dos campi do IFBA e cria um ranking das eficiências. Os resultados demonstraram a existência de diferenças significativas de eficiência entre as unidades. Apontaram a necessidade do aumento do número de concluintes dos cursos para alcançarem o patamar do campus de Eunápolis que ocupa a fronteira de eficiência.

Palavra-chave: Eficiência. Recursos públicos. Educação. IFBA.

Abstract: It was analyzed the relative efficiency of IFBA's campi as the main purpose of this research. In addition define the efficiencies in offering initial and continuing training courses and make recommendations for the improvement in the efficiency of the units analyzed. The analysis method applied is the quantitative one, by means of Data Envelopment Analysis. It analyzes only 6 IFBA campi and establishes an efficiency ranking. The results evinced the existence of significant efficiency imbalance between units. They pointed to the need to increase the number of graduates of the courses in order to reach the level of Eunápolis campi which is at the efficiency frontier.

Key-word: Efficiency. Public resources. Education. IFBA.

\footnotetext{
${ }^{1}$ Mestre em Administração pela Universidade Federal da Bahia (UFBA).E-mail: fabiolemosmota@bol.com.br ${ }^{2}$ Doutor em Administração pela Universidade Federal da Bahia (UFBA). Professor da Escola de Administração da UFBA, atuando na graduação e na pós-graduação stricto sensu. E-mail:robertobrazileiro@gmail.com

${ }^{3}$ Doutora em Ciências da Comunicação, professora permanente no Programa de Pós-graduação em Educação e no Programa de Pós-graduação em Tecnologias, Comunicação e Educação, Universidade Federal de Uberlândia (UFU).E-mail: adriana.omena@ufu.br
} 
Résumé: L'efficacité relative des campus IFBA comme objectif principal de cette recherche a été analysée. En plus, l'efficacité des offres de formation initiale et continue a été définie et des recommandations ont été formulées pour améliorer l'efficacité des unités analysées.La méthode d'analyse utilisée est quantitative, en utilisant la technique d'analyse de l'enveloppe de données. Il analyse seulement 4 cycles de campus IFBA et a crié un classement des efficiences. Les résultats ont démontré l'existence de différences d'efficacité significatives entre les unités. Ils ont souligné la nécessité d'augmenter leurs nombre de diplomês des cours pour atteindre le campus de Eunápolis, qui occupent le sommet de l'échelle em niveau l'efficacité.

Mots clés: Efficacité. Ressources publiques. Éducation. IFBA.

\section{Introdução}

Historicamente, a eficiência da administração pública brasileira não era algo essencial, devido ao reduzido papel que o Estado desempenhava. Só a partir do século XX, com a expansão do seu campo de atuação tanto na área social como na área econômica, se transformando num Estado social e econômico, é que a questão da eficiência se torna indispensável (PEREIRA, 1996).

A educação obteve um grande volume de investimentos do governo federal nos últimos doze anos que antecederam ao atual governo. Há exemplo dos planos de expansão dos Institutos Federais de Educação Técnica e do Programa de Apoio a Planos de Reestruturação e Expansão das Universidades Federais (Reuni). Porém, no ano de 2016 foi aprovada a Emenda Constitucional n. ${ }^{\circ}$ 95, que alterou a Constituição brasileira de 1988 para instituir o Novo Regime Fiscal ou o chamado Teto do Gasto Públicos, com congelamento e corte significativo de recursos inclusive para a educação (BRASIL, 2016). Desta forma, mais do que nunca, estes escassos recursos precisam ser geridos com eficiência e ser alvo de avaliação constante para garantir o atendimento aos fins a que se destinam - a melhoria ou pelo menos manutenção da qualidade da educação pública.

A Lei de Diretrizes e Bases da Educação Nacional (LDB) define que a educação profissional e tecnológica integra-se aos diferentes níveis e modalidades de educação abrangendo, entre outros cursos, o de formação inicial e continuada (FIC) (BRASIL, 1996). Este tipo de curso que inclui a capacitação, o aperfeiçoamento, especialização e atualização em todos os níveis de escolaridade, pode ser organizado em itinerários formativos para o 
desenvolvimento de aptidões para a vida produtiva e social. Também terão de ter carga horária mínima de cento e sessenta horas para a formação inicial, para além das etapas posteriores de formação continuada (BRASIL, 2004).

No ano de 2017, duzentas e dezoito unidades de ensino da Rede Federal de Educação Profissional, Científica e Tecnológica ofertaram cursos FIC na área de Desenvolvimento Educacional, disponibilizando um total de noventa e oito mil seiscentas e sessenta vagas. Destas, porém, foram realizadas aproximadamente sessenta e oito mil matrículas gerando o número de cinquenta e um mil e quatrocentos ingressantes, mas resultando em apenas algo próximo de trinta e sete mil concluintes (PLATAFORMA NILO PEÇANHA, 2018). Números que sugerem, uma baixa capacidade do sistema em ocupar as vagas ofertadas quarenta por cento não foi ocupada - reter os alunos e garantir a conclusão dos cursos - que foi de apenas trinta e sete por cento.

O Instituto Federal de Educação, Ciência e Tecnologia da Bahia (IFBA) é uma autarquia vinculada à Secretaria de Educação Profissional e Tecnológica (SETEC) do Ministério da Educação (MEC) e possui como atividades principais o ensino, a pesquisa e a extensão na educação básica e superior. Com a expansão da rede, saltou de quatro unidades de ensino para vinte e um campi, um núcleo avançado e reitoria em 2018, distribuídas em todas as regiões do Estado da Bahia.

O objetivo geral desta pesquisa é analisar a eficiência relativa de determinados campi do IFBA em termos de resultados alcançados em função dos recursos alocados. Os objetivos específicos são definir as eficiências dos campi em oferecer cursos FIC com os recursos disponíveis. Em seguida analisar relativamente a eficiência destas unidades acadêmicas do IFBA e por fim, fazer recomendações para a melhoria da eficiência e aperfeiçoamento das unidades analisadas.

Foram avaliados apenas quatro dos vinte e um campi do IFBA: Eunápolis, Lauro de Freitas, Santo Antônio de Jesus e Vitória da Conquista. O critério de escolha dos campi foi possuir cursos FIC do eixo tecnológico Desenvolvimento Educacional e Social - um dos treze eixos constantes no catálogo do Conselho Nacional de Educação (CNE).

\section{Avaliação Institucional}

Hadji (2001) defende que avaliar não se trata de simplesmente medir, observar ou emitir juízo de valor. É afirmar como as expectativas foram realizadas. A avaliação é uma leitura da realidade com a utilização de um método. 
Para avaliar algo, primeiro é preciso determinar o seu referente, ou seja, os critérios e expectativas. Os critérios irão definir o que esperar do objeto avaliado. Esse referente da avaliação deve ser determinado a partir de um conjunto de expectativas a serem considerados para cada avaliação. Como esta escolha e triagem são questionáveis, os referentes são sempre discutíveis. Mas, sem que isto seja um obstáculo à avaliação, sempre é possível questionar o quão legítimo é o referente (HADJI, 2001).

O conceito de eficiência aqui adotado é de eficiência econômica, também chamada de Ótima ou Eficiência de Pareto. Nesse conceito, a eficiência se dá quando pelo menos um agente melhora sem que com isso outro piore. No cálculo da eficiência econômica, os coeficientes de produção se relacionam de forma que é possível uma compensação da diminuição de um pelo aumento de outro. Porém, esta relação nem sempre é possível para alguns coeficientes (PARETO, 1996, p.257). O bem estar máximo ocorre quando não existe outro estado que se possa aumentar o bem estar de um indivíduo, sem que isto implique na redução do bem estar dos demais. Em resumo, não é possível melhorar a situação de um sem prejudicar a situação dos outros (PARETO, 1996, p.13).

A avaliação da eficiência de políticas públicas - e em especial de instituições de ensino - tem sido objeto de inúmeras pesquisas no Brasil.

Belloni (2000), por exemplo, avaliou 33 universidades federais brasileiras com a Análise Envoltório de Dados (DEA). Foram identificadas 6 universidades eficientes que deram origem a metas de eficiência para as outras 27 consideradas ineficientes. Concluiu-se que a eliminação dos focos de ineficiência técnica identificados geraria um aumento de 18\% na quantidade de formandos e $10 \%$ na publicação de artigos, com o mesmo quadro de docentes (BELLONI; 2000).

\section{Análise Envoltória de Dados}

Nesta pesquisa de avaliação, será analisada apenas a eficiência. O principal método de análise utilizado foi o quantitativo, com o uso da DEA. Esta técnica consiste na comparação de entradas inputs e saídas outputs no sistema analisado. Foi utilizado também a pesquisa documental para obtenção dos valores das variáveis utilizadas.

Como forma de medir a eficiência relativa entre unidades produtivas foi criada a DEA, realizada geralmente por software, os dados de input e output são tratados para avaliar a eficiência relativa de determinada unidade de análise (BELLONI; BELLONI, 2003). A 
eficiência calculada é dita relativa porque as unidades - que na presente pesquisa são campus), chamadas nesta técnica de Decision Making Units (DMU), tem seu desempenho medido pela comparação de seus resultados e seus insumos, com os resultados e insumos de outras unidades homogêneas da amostra, ou seja, que recebem o mesmo conjunto de recursos para produzir o mesmo conjunto de resultados, por meio de processos similares.

Para Lins e Meza (2000), as unidades consideradas eficientes representam uma fronteira de eficiência (Eficiência de Pareto) e serão classificadas como de eficiência 1 ou $100 \%$, permitindo a comparação entre as unidades. As unidades que estiverem próximas a esta fronteira serão ditas eficientes, enquanto a distância das demais unidades até esta fronteira irá determinar suas ineficiências. A fronteira de eficiência se constituirá em meta para cada plano considerado ineficiente, determinando ações e estratégias para aumentar a produtividade da unidade. A DEA não recorre, assim, a parâmetros externos ao sistema, ou seja, é uma técnica não paramétrica.

A forma original de cálculo da eficiência da DMU segundo Banker, Charnes e Cooper (1984) é a seguinte:

A eficiência da DMU é resultado da razão entre a quantidade de cada output da DMU multiplicado pelo peso outorgado ao output, e a quantidade de cada input multiplicado pelo peso a ele também outorgado.

O ranking de eficiência é calculado pela razão entre a eficiência de determinada DMU e a eficiência da DMU mais eficiente. A determinação dos pesos de cada variável dependerá de critérios para sua definição. Para eliminar a subjetividade, cada DMU deve adotar os pesos que maximizem essa razão, tendo o cuidado de manter o resultado entre o e 1 (SOARES DE MELO; MEZA et al, 2005).

Existem diferentes modelos de DEA, um modelo clássico é o denominado CCR. Este modelo criado por Charnes, Cooper e Rhodes (1978), utiliza retornos constantes de escala, ou seja, qualquer variação no input causará instantânea modificação proporcional do output. $O$ CCR orientado a output, maximiza os resultados (output), mantendo inalteradas as entradas (input).

Este método é o que mais se aplica às características do modelo aqui desenvolvido, visto que os recursos da educação (financeiro, professores, técnicos etc.) precisam sim aumentar e não serem reduzidos. 


\section{Procedimentos Metodológicos}

A eficiência será determinada por meio da aplicação da DEA, em dois passos: seleção das variáveis e cálculo do indicador da eficiência.

O primeiro passo é a seleção do conjunto de variáveis mais representativas dos recursos utilizados pela instituição (inputs) e outro conjunto dos resultados alcançados (outputs). Os dados coletados são referentes ao ano de 2017.

Uma lista de possíveis variáveis de input e output foi levantada através de revisão de literatura. O número destas variáveis, assim como de DMUs, deve permitir que haja alguma distinção entre as DMUs e não tenham unidades em excesso na fronteira de eficiência, o que prejudicaria a capacidade da DEA em separar unidades eficientes das ineficientes, conforme apregoam Soares de Melo, Meza e outros (2005). Desta forma, o primeiro filtro foi a disponibilidade dos dados - de fonte segura - referentes a cada variável, no caso, a Plataforma Nilo Peçanha.

Dada a limitação do número de variáveis em relação ao número de DMUs, imposta pela DEA - o número das variáveis em cada aplicação deve ser no máximo a metade do número de DMUs (ESTELLITA LINS; MOREIRA, 1999 apud BRAZ, 2005) - como o número de DMUs é de quatro, foram utilizadas no máximo duas variáveis na aplicação da DEA, distribuídas entre input e output.

Quadro 1: Variáveis Selecionadas.

\begin{tabular}{|l|l|}
\hline \multicolumn{1}{|c|}{ Inputs } & \multicolumn{1}{c|}{ Outputs } \\
\hline $\begin{array}{l}\text { Número de Vagas } \\
\text { oferecidas nos Cursos FIC }\end{array}$ & $\begin{array}{l}\text { Número de Concluintes nos } \\
\text { Cursos FIC }\end{array}$ \\
\hline
\end{tabular}

\section{Aplicação da Técnica DEA}

O software Sistema Integrado de Apoio a Decisão (Siad), versão 3.0 - programa com modelos de DEA (ANGULO at al, 2005) - foi escolhido para realização dos cálculos matemáticos que irão gerar os índices de eficiência das DMUs, os pesos dos inputs, a folga entre o input da DMU e seu benchmark e valores alvo para alcance da eficiência 1 ou 100\%.

Da utilização do software SIAD, com os dados das variáveis do apêndice A, foram geradas as seguintes eficiências: 
Quadro 2: Índice de Eficiência da DMU (ranking).

\begin{tabular}{|l|c|}
\hline \multicolumn{1}{|c|}{ DMUs } & Índice \\
\hline Eunápolis & 1,00 \\
\hline Lauro de Freitas & 0,98 \\
\hline Santo Antônio de Jesus & 0,57 \\
\hline Vitória da Conquista & 0,46 \\
\hline
\end{tabular}

Verifica-se a ocorrência de uma excelente distinção entre as unidades, como recomendado por SENRA at al (2007), com apenas uma unidade na fronteira de eficiência $(1,0)$. Tornando este modelo apropriado para a análise. Passamos agora aos pesos atribuídos às variáveis pelo software para maximizar as eficiências.

Quadro 3: Pesos Atribuídos às Variáveis pelo SIAD.

\begin{tabular}{|l|c|c|}
\hline \multicolumn{1}{|c|}{ DMUs } & Pesos Vagas & $\begin{array}{c}\text { Pesos } \\
\text { Concluintes }\end{array}$ \\
\hline Eunápolis & 0,01000000 & 0,01041667 \\
\hline Lauro de Freitas & 0,02285714 & 0,02380952 \\
\hline $\begin{array}{l}\text { Santo Antônio de } \\
\text { Jesus }\end{array}$ & 0,01684211 & 0,01754386 \\
\hline Vitória da Conquista & 0,04363636 & 0,04545455 \\
\hline
\end{tabular}

Como visto no método, a DEA atribuiu pesos às variáveis de forma a potencializar a eficiência da DMU. Verifica-se que todas as DMUs tiveram peso maior para a variável Concluintes. Afinal, elas precisam de bons indicadores nesta variável. Em compensação tiveram pesos menores para Vagas, indicando que não contribuem para a eficiência destas DMUs. Apesar de ser importante o aumento da oferta de vagas na educação, se não forem preenchidas, se darão perdas de recursos investidos.

\section{Alvos}

O software SIAD faz sugestões de alvos a serem alcançados para melhoria da eficiência. Com exceção do campus Eunápolis e Lauro de Freitas - que tem discreta indicação de aumento de concluintes, os demais comprometem a eficiência devido ao baixo número de concluintes. O output precisa de incremento significativo em dois campus para que a eficiência relativa seja alcançada. Deve aumentar em 77\% em Santo Antônio de Jesus, já Vitória da Conquista precisaria de $118 \%$ de aumento. 
A DMU Eunápolis foi o benchmark do modelo adotado. Ela representa a fronteira de eficiência onde as demais DMUs foram projetadas. E seus indicadores foram utilizados para determinação dos alvos a serem atingidos para que todas se tornem eficientes.

Como ilustrado no ranking decrescente de eficiência dos campus, existe uma significativa diferença de desempenho entra as DMUs. Considerando as variáveis adotadas no modelo escolhido.

Eunápolis ocupa a fronteira de eficiência e Lauro de Freitas demonstra proximidade desta fronteira. Já o campus Vitória da Conquista é o de menor eficiência relativa com grande discrepância em relação aos demais.

Fica clara a possibilidade de aumento da eficiência da maioria dos campus do IFBA. Sendo a tarefa de aumentar os concluintes o ponto mais sensível desta empreitada.

\section{Conclusão}

Do objetivo principal desta pesquisa, de analisar a eficiência relativa de determinados campi do IFBA em termos de resultados alcançados em função dos recursos alocados, pode-se concluir que o IFBA vive realidades muito distintas entre suas unidades em termos de eficiência. Onde um campus como Eunápolis possuiu eficiência ótima enquanto outro, como Vitória da Conquista, possui eficiência quase cinquenta por cento menor, para as variáveis analisadas. Vale frisar que estes campus foram fundados na mesma época de 1994 e 1995 respectivamente.

Já o objetivo específico de definir as eficiências dos campi em oferecer cursos FIC com os recursos disponíveis teve como resultado os campi de Eunápolis e Lauro de Freitas como os mais eficientes. Onde Lauro de Freitas teve a eficiência próxima à fronteira estabelecida pelo primeiro, dito eficiente. Os campi de Santo Antônio de Jesus e Vitória da Conquista demonstraram baixa eficiência.

As recomendações que possivelmente levarão os campus ao alcance da eficiência começam pela investigação e diagnóstico das causas da evasão nos cursos FIC. Tal façanha pode ter o auxílio de medidas como: acompanhamento dos números da evasão, levantamento das causas do abandono, avaliação e categorização das principais causas e fortalecimento da assistência ao aluno. 
As vagas ociosas representam um desperdício de recursos. O recurso público é resultado de impostos que, no caso brasileiro, é concentrado na renda das pessoas e por isso precisa ser utilizado com eficiência, sem desperdício.

Cabe ressaltar que este trabalho não tem a presunção de fechar definitivamente o diagnóstico sobre as eficiências das unidades avaliadas, pois os cursos FIC não são as únicas ou mesmo as principais atividades de algumas unidades. Tampouco está o método imune a questionamentos, até por não esgotar todo o universo de fatores passíveis de influenciar a eficiência. Desta forma, os resultados devem ser apreciados como mais uma ferramenta de apoio à decisão e precisam ser interpretados com cautela.

\section{Referências}

ANGULO MEZA, L.; BIONDI NETO, L.; SOARES DE MELLO, J.C.C.B.; GOMES, E.G. ISYDS - Integrated System for Decision Support (SIAD - Sistema Integrado de Apoio à Decisão): a software package for data envelopment analysis model. Pesquisa Operacional, v. 25, (3), p. 493-503, 2005

ANGULO MEZA, L.; BIONDI NETO, L.; SOARES DE MELLO, J.C.C.B.; GOMES, E.G.; COELHO, P.H.G. Free software for decision analysis: a software package for data envelopment models. In: 7th International Conference on Enterprise Information Systems - ICEIS 2005, v. 2, p. 207-212.

BANKER, R.D.; Charnes, A.; Cooper, W.W. Some models for estimating technical and scale inefficiencies in Data Envelopment Analysis. Management Science. v. 30, n. 9, 1984.

BELLONI, Isaura; BELLONI, José Â. Questões e Propostas para uma Avaliação Institucional Formativa In: FREITAS, Luiz C. (org.) Avaliação de escolas e universidades. São Paulo: Komedi, 2003. p. 9-54.

BELloni, José Â. Uma Metodologia de Avaliação da Eficiência Produtiva de Universidades Federais Brasileiras. 2000. 246 p. Tese (Doutorado em Engenharia de Produção) Universidade Federal de Santa Catarina, Florianópolis.

BRASIL. Lei $n^{\circ}$ 9.394, de 20 de dezembro de 1996. Estabelece as diretrizes e bases da educação nacional. Diário Oficial da União, Brasília, DF, 23 dez.1996.

Decreto $\mathrm{n}^{\mathrm{o}} 5.154$, de 23 de julho de 2004 . Regulamenta o $\S 2^{\circ}$ do art. 36 e os arts. 39 a 41 da Lei $n^{\circ}$ 9.394, de 20 de dezembro de 1996, que estabelece as diretrizes e bases da educação nacional, e dá outras providências. Diário Oficial da União, Brasília, DF, 26 jul. 2004.

Emenda Constitucional $\mathrm{n}^{\circ}$ 95, de 15 de dezembro de 2016. Altera o Ato das Disposições Constitucionais Transitórias, para instituir o Novo Regime Fiscal, e dá outras providências. Diário Oficial da União, Brasília, DF, 15 dez. 2016. 
BRAZ, Gisele Figueiredo. Aplicação de um Método Quantitativo e Comparado, A Análise de Envoltória de Dados (DEA), para Avaliação do Desempenho dos Departamentos Acadêmicos da Universidade Estadual de Montes Claros. 109 p. Dissertação (Mestrado em Engenharia de Produção). Escola de Engenharia da Universidade Federal de Minas Gerais, Belo Horizonte, 2005.

CHARNES, A.; COOPER, W.W.; RHODES, E. Measuring the efficiency of decision-making units. European Journal of Operational Research, v. 2, p. 429-444, 1978. Disponível em: http://www.utdallas.edu/ ryoung/phdseminar/CCR 1978.pdf. Acessado em: 19/12/2012.

HADJI, Charles. A avaliação desmistificada. Porto Alegre: Artemed, 2001. 136 p.

LINS, Marcos Pereira Estellita; MEZA, Lidia Angulo. Análise envoltória de dados e perspectivas de integração no ambiente do apoio à decisão. Rio de Janeiro: Coppe/UFRJ, 2000.

LYRA, Ricardo L. W. C. Análise Hierárquica dos Indicadores Contábeis sob a Óptica do Desempenho Empresarial. 171 p. Tese (Doutorado em Controladoria e Contabilidade) Faculdade de Economia, Administração e Contabilidade de São Paulo, São Paulo, 2008.

PARETO. Manual de Economia Política. Tradução de João Guilherme Vargas Netto. São Paulo: Nova Cultura, 1996. 333 p. (Coleção Os Economistas). Versão em inglês de Ann S. Schiwier, da versão francesa revisada de Giard Briére do original em italiano.

PEREIRA, Carlos Bresser. Da Administração Pública Burocrática à Gerencial. Revista do Serviço Público, 1996, Vol. 47(1) janeiro-abril.

PLATAFORMA NILO PEÇANHA. PNP 2018 (Ano Base 2017). Disponível em: https://www.plataformanilopecanha.org/ Acessado em: 27/06/2018.

SENRA, L. F. A. de Castro; NANCI, Luiz Cesar; MELlO, J. C. C. B. Soares de; MEZA, Lidia Ângulo. Estudo sobre métodos de seleção de variáveis em DEA. Pesquisa Operacional, vol.27, no.2, Rio de Janeiro, May/Aug. 2007.

SOARES DE MELLO, J.C.C.B; MEZA, L.A.; GOMES, E.G.; BIONI NETO, L. .Curso de Análise de Envoltória de Dados. In: Anais XXXVII Simpósio Brasileiro de Pesquisa Operacional (SBPO). Gramado, RS, 2005.

\section{Apêndice}

A - Valores das Variáveis por Unidade.

\begin{tabular}{|l|c|c|}
\hline DMUs & Vagas & Concluintes \\
\hline Eunápolis & 100 & 96 \\
\hline Lauro de Freitas & 44 & 42 \\
\hline Sto Antônio de Jesus & 105 & 57 \\
\hline Vitória da Conquista & 50 & 22 \\
\hline
\end{tabular}

Fonte: Plataforma Nilo Peçanha (2018) 